\title{
Which is Better in Laborers? A Comparison Between Open and Micro Endoscopic Discectomy
}

\author{
Hitesh N. Modi ${ }^{1,2}$, Tushar Kunder ${ }^{1}$, Neel Bhavsar ${ }^{1}$, Pankaj R. Patel ${ }^{1}$
}

\section{Abstract}

Introduction: None of the studies compared the results of open lumbar discectomy (OLD) and microendoscopic discectomy (MED) surgeries in laborers. The aim of this study was to compare the clinical and functional impact of OLD versus MED surgery in laborers to find out which is better.

Materials and Methods: This retrospective study was performed in 91 laborers ( 54 males and 37 females) who underwent OLD $(\mathrm{n}=41)$ versus MED ( $\mathrm{n}=50)$ for the single- or double-level lumbar disc herniation (LDH). All patients were operated at a single institute after a failure of conservative trial for 6 weeks. Patients with associated severe disc degeneration, stenosis, instabilities, or other pathologies were excluded from the study. The clinical results were evaluated with Oswestry Disability Index (ODI), visual analog score (VAS), and duration of return back to work.

Results: The average age of the study group was $39.8 \pm 12.1$ years. Average follow-up was $50.2 \pm 13.9$ months. The entire study group comprised manual labor work such as farming or loading work with an average income of US $\$ 53.6 \pm 14.6$ (approximately INR 4000) per month. The patients belonged to low socioeconomic status as per modified Kuppuswamy scale. The post-operative VAS scores were significantly reduced in both MED (7.6-2.0) and open discectomies (7.2-2.1). Improvement ODI scores also showed similar trends for MED (57.3-20.6) and for open discectomies (55.1-20.1). Average duration to return to work was significantly less in the MED group in comparison to the OLD group ( 18.0 vs. 25.5 days). There were total 4 (4.4\%) complications perioperatively. There were one superficial wound infection in the OLD and one dural tear in the MED group. Both were managed conservatively. There was one patient from each group having recurrent disc herniation that was managed conservatively. There were one patient from the MED and two patients from the OLD group who could not return to their previous work or had to modify their work due to back pain.

Conclusion: Although clinical improvement after discectomy surgery in laborers is similar, MED is a promising alternative to OLD in laborers with respect to return to work earlier. Such studies may further throw light in differential management of laborer population with MEDs versus OLD.

Keywords: Lumbar disc herniation; Laborers; Discectomy technique; Early return to work.

\section{Introduction}

Low back pain is an emerging world public health issue plaguing the population in general and a burden on the healthcare industry. According to the literature, lifetime prevalence for low back pain is $84 \%$ and for chronic low back pain is $23 \%$,

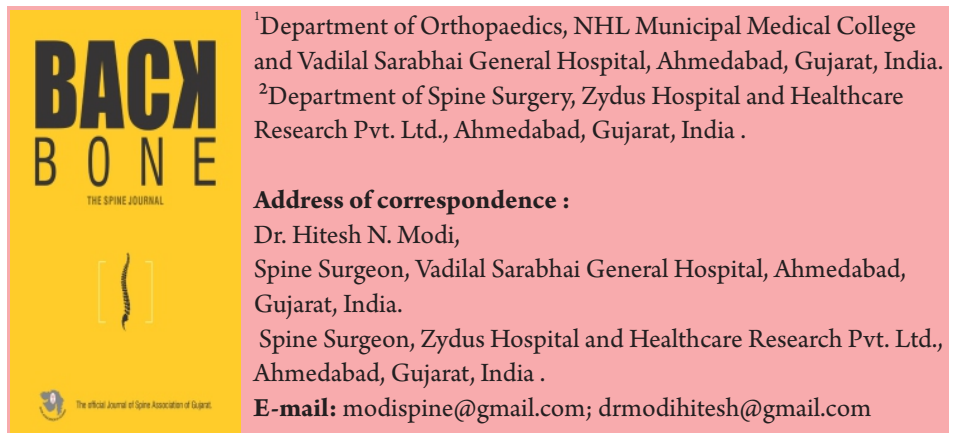

with $11-12 \%$ of the population being disabled due to chronic low back pain [1]. Lumbar disc herniation (LDH) is one of the most common spinal degenerative disc disorders associated with low back pain and radiculopathy $[2,3]$. Some of the studies have reported the association of lumbar disc disease with heavy manual labor such as dockworkers who are involved in lifting heavy loads [4]. Mechanical disorders of the lower back are thought to result from a combination of constitutional weakness and physical stress to the spine. Lumbar discectomy for lumbar disc disease is about $70-90 \%$ successful in disc prolapse $[5,6]$. Traditional open surgery or microdiscectomy, which is known as open lumbar discectomy (OLD), is still the gold standard for surgical intervention for LDH. Lumbar microendoscopic discectomy (MED) is also a 


\begin{tabular}{|c|c|c|c|}
\hline Group & OLD group & MED group & Total \\
\hline No. of patients & 41 & 50 & 91 \\
\hline single-level disc & 31 & 40 & 71 \\
\hline Double-level disc & 10 & 10 & 20 \\
\hline Average age (years) & $37.7 \pm 7.8$ & $41.5 \pm 14.5$ & $39.8 \pm 12.1$ \\
\hline Male/female & $22 / 19$ & $32 / 18$ & $54 / 37$ \\
\hline Average FU (months) & $50.3 \pm 14.4$ & $50.2 \pm 13.7$ & $50.2 \pm 13.9$ \\
\hline
\end{tabular}

well-established procedure with low surgical morbidity and similar success rates [7]. The standard surgical treatment of $\mathrm{LDH}$ has been open discectomy but there has been a trend toward minimally invasive procedures. MED for the LDH is a well-reported surgical technique, which is increasingly popular in the past few years $[5,6]$. It is becoming an effective alternative to standard OLD as almost all types of disc herniation can be treated with MED [7].

There are different post-operative protocols followed up worldwide after the discectomy procedures. Based on the recommendation of the North American Spine Society (NASS), most people with jobs that are not physically challenging can return to work in 2-4 weeks or less. According to Rompe et al., what is more important as a prognostic criteria for patient selection for discectomy are the strongest possible objective success parameter, that is, return to work, after a surgery in their series with 9-year follow-up [8]. Yadav et al. reported that MED entailed shorter hospital stay, less morbidity, earlier return to work, less blood loss, and shorter operation time comparing to OLD [5]. However, which operative technique facilitates encouraging results with early return to work in manual workers or laborers who are involved in daily work such as farming or manual labor is not reported in the literature. As these people in developing countries have to rely on their daily earnings for their daily living, and therefore, it is important for them to regain their functionality as soon as possible. Primary aim of our study was to compare operative outcomes between two techniques OLD and MED in patients with LDH and involved in labor work for their daily living. Our purpose was to find out which technique should be implemented in such patients for LDH that helps them earning and returning to productivity.

\section{Materials and Methods}

This retrospective analytical study was conducted in patients who were operated for LDH between January 2014 and December 2017. There were 91 unskilled laborers comprising 54 men and 37 women belonging to low socioeconomic status according to the modified Kuppuswamy scale included in the study [9]. The average age of the study population was $39.8 \pm$ 12.1 years (range, $21-78$ years). Operating surgeon randomly

\begin{tabular}{|l|c|c|c|}
\hline $\begin{array}{l}\text { Table 2: Pre-operative and post-operative VAS and ODI scores in both } \\
\text { groups }\end{array}$ \\
\hline \multicolumn{1}{|c|}{ Group } & OLD group & MED group & Total \\
\hline Pre-operative VAS & $7.2 \pm 1.1$ & $7.6 \pm 1.4$ & $7.4 \pm 1.3$ \\
\hline Post-operative VAS & $2.1 \pm 0.9$ & $2.0 \pm 0.8$ & $2.1 \pm 0.8$ \\
\hline Pre-operative ODI & $55.1 \pm 9.7$ & $57.3 \pm 8.6$ & $56.3 \pm 9.1$ \\
\hline Post-operative ODI & $21.0 \pm 8.9$ & $20.6 \pm 8.8$ & $20.8 \pm 8.8$ \\
\hline
\end{tabular}

had selected patients for OLD $(n=40)$ or MED $(n=45)$ in a government institute (Table 1). All patients were operated by a single surgeon and followed in outpatient clinic regularly postoperatively for a minimum period of 2 years. There were 71 and 20 patients with single-level and double-level disc herniation, respectively (Table 1). Patients who had persistent radicular symptoms or worsening of symptoms after 6 weeks of conservative treatment, along with straight leg raising (SLR) and crossed straight leg raising tests (CSLR) positive with the absence of corresponding neurological deficits, were included in the study. Patients with severe disc degeneration, canal stenosis, spinal instabilities, cauda equina syndrome, or other pathologies were excluded from the study. All patients were selected for surgery from the outpatient department (OPD) after failure of conservative trial. Patients who were admitted and operated through the emergency room (ER) with the same problem were not included in the study.

All patients were followed preoperatively and postoperatively at an interval of 6 weeks, 3 months, 6 months, 1 year, and 2 years after surgery. The clinical results were analyzed and compared preoperatively and postoperatively using the visual analog score (VAS) and Oswestry Disability Index (ODI) questionnaire which was duly noted down by the patients with assistance from resident doctors preoperatively and at 6 weeks and at the final follow-up postoperatively. MacNab's criteria postoperatively, intraoperative estimated blood loss (EBL), operation time, hospital stay, and duration of return back to work were also measured and compared between the OLD and MED groups. Paired and unpaired t-tests were used to find out statistically significant differences (SPSS, version 12, Chicago, Illinois); and $\mathrm{P}<0.05$ kept to be significance value.

\section{Results}

Average follow-up was $50.2 \pm 13.9$ months. In both groups, there was significant improvement in pre-operative VAS score of $7.2 \pm 1.1$ to post-operative score of $2.1 \pm 0.9$ in the OLD group $(\mathrm{P}<0.0001$, paired $\mathrm{t}$-test $)$ and pre-operative $7.6 \pm 1.4$ to post-operative $2.0 \pm 0.8$ in the MED group $(\mathrm{P}<0.001$, paired $\mathrm{t}$ test) (Table 2). Similarly, the improvement in the ODI scores was significant from pre-operative $55.1 \pm 9.7$ to post-operative $21.0 \pm 8.9$ in the OLD group $(\mathrm{P}<0.001$, paired t-test $)$ and pre- 


\begin{tabular}{|c|c|c|c|c|c|}
\hline \multicolumn{6}{|c|}{ Table 3: MacNab's criteria in both groups } \\
\hline MacNab's criteria & \multicolumn{2}{|c|}{ Excellent } & Good & Fair & Poor \\
\hline OLD\% $(n)$ & \multicolumn{2}{|c|}{$63.4(26)$} & $29.3(12)$ & $4.9(2)$ & $2.4(1)$ \\
\hline $\operatorname{MED} \%(n)$ & \multicolumn{2}{|c|}{$68(34)$} & $22(11)$ & $8(4)$ & $2(1)$ \\
\hline \multicolumn{6}{|c|}{$\begin{array}{l}\text { Table 4: Average operation time, blood loss, hospital stay, and } \\
\text { return to work in both groups }\end{array}$} \\
\hline \multicolumn{2}{|l|}{ Group } & \multicolumn{2}{|c|}{ OLD group } & MED group & Total \\
\hline \multicolumn{2}{|c|}{ Operation time (min) } & \multicolumn{2}{|c|}{$62.4 \pm 18.7$} & $64.0 \pm 19.5$ & $63.3 \pm 19.0$ \\
\hline \multicolumn{4}{|c|}{ Estimated blood loss (mL) $66.1 \pm 24.1$} & $32.1 \pm 12.5$ & $47.4 \pm 25.1$ \\
\hline \multicolumn{2}{|c|}{ Hospital stay (days) } & \multicolumn{2}{|c|}{$5.0 \pm 0.9$} & $2.5 \pm 0.6$ & $3.6 \pm 1.4$ \\
\hline \multicolumn{2}{|c|}{ Return to work (days) } & \multicolumn{2}{|c|}{$25.5 \pm 7.3$} & $18.0 \pm 4.0$ & $21.4 \pm 6.8$ \\
\hline
\end{tabular}

operative $57.3 \pm 8.6$ to post-operative $20.6 \pm 8.8$ in the MED group $(\mathrm{P}<0.0001$, paired t-test) (Table 2$)$. MacNab's criteria postoperatively for the OLD and MED groups were significant showing more than $90 \%$ of patients had excellent and good outcome, which was similar and comparable in both groups (Table 3$)$. Hospital stay was significantly $(\mathrm{P}<0.05)$ higher in the OLD group than the MED group ( $5.0 \pm 0.9$ vs. $2.5 \pm 0.6$ days; P $<0.05$, Fisher's test). Similarly, estimated blood loss during operation was also higher in the OLD group than the MED group $(66.1 \pm 24.1 \mathrm{~mL}$ vs. $32.1 \pm 12.5 \mathrm{~mL}, \mathrm{P}<0.05$, Fisher's test). Average operation time, however, was similar in both groups (62.4 $\pm 18.7 \mathrm{~min}$ vs. $64.0 \pm 19.5 \mathrm{~min}, \mathrm{P}>0.05$, Fisher's test). However, there was significantly higher period for returning to work in the OLD group than the MED group (25.5 \pm 7.3 days vs. $18.0 \pm 4.0$ days, $P<0.05$, Fisher's test) from the day of discharge from the hospital (Table 4). If we add further longer hospital stay in the OLD group, this gap between two groups is even wider.

There were total 4 patients (4.4\%) with complications in perioperative period. There was one superficial wound infection in the OLD group and one dural tear in the MED group. Both were managed conservatively. Patient with superficial wound infection had Staphylococcus aureus growth on culture, which was treated with 1 week of intravenous and 2 weeks of oral antibiotics with local dressing without any further sequel. One patient with intraoperative dural leak from the MED group was treated conservatively with packing ofleak with local fat graft and gelfoam. Patient was given bed rest for 5 days before mobilization. There was one patient from each group having recurrent disc herniation that was managed conservatively with anti-inflammatory medicines and nerve root block. Both patients presented with recurrent disc herniation at least 3 months after the index surgery. Symptoms were relieved with conservative treatment in both patients within 6 weeks period. There were one patient from MED and two patients from the OLD group who could not return to their previous work or had to modify their work due to back pain.

\section{Discussion}

$\mathrm{LDH}$ is the most common cause of low back and leg pain. It affects significantly the quality of the life [10]. If the patients who have serious or typical symptoms cannot be healed more than 6-8 weeks by conservative treatment, operation should be indicated as the only one effective method [11]. Two main surgical methods are used commonly: OLD and MED. OLD has been proved to be effective and also was widely used. As a new minimally invasive operative method, MED was initially introduced by Smith and Foley in 1997 [12]. The advantages of MED over OLD are small incision, better cosmetic appearance, early ambulation, less post-operative pain, less intraoperative blood loss, shorter hospital stay, and shorter time to return to work $[13,14,15]$. MED is less traumatizing to the skin and the paraspinal muscles, resulting in reduced fibrosis of the canal [16]. These all advantages in MED indirectly lead to early return to work and thereby reducing the financial loss. However, it is really important in laborers as they need to earn daily living for their family and therefore even a single day of jobless would count significantly to their living. We have included 91 individuals who were involved in manual labor work or farm working for their daily earning and their financial status was in lower socioeconomic class by modified Kuppuswamy classification [9]. Our study focused on this issue that was not reported before.

The ODI and VAS, preoperatively and postoperatively in both the study groups, were significant and similar, these results of our study were similar to other studies comparing OLD and MED. Improvements in ODI postoperatively in both MED and OLD groups were similar when compared across studies in India $[17,18]$. However, Bock et al. also reported that the postsurgical analgesic consumption is significantly less if a tubular retractor is inserted through a transmuscular approach [19]. The reason behind is that the open surgery includes extensive retraction and dissection of paraspinal muscles, longer operative time, longer incisions, and bone resection [20]. Garg et al. in their prospective randomized trial comparison between OLD and MED in 112 patients mentioned that both methods are equally effective in relieving radicular pain and there is similar improvement in ODI as well as complications [17]. However, MED entailed shorter hospital stay, less morbidity, and earlier return to work as an advantage over OLD. Kulkarni et al. reported their outcome in 188 consecutive patients who were operated with tubular retractor system (MED) for LDH [6]. Their mean operation time was $50 \mathrm{~min} /$ per level and average blood loss was $30 \mathrm{~mL}$. Improvement in VAS and ODI score postoperatively was significant. They have encouraged their patients to return to their work in 3 weeks. Our results are quite similar to the published reports suggesting similar clinical outcome in both OLD and MED. There was a significant improvement in VAS 
and ODI score in both groups in our series of 91 patients which proves that MED is equally effective as OLD in clinical results. Operation time in our series was $62.4 \pm 18.7 \mathrm{~min}$ in the OLD group and $64.0 \pm 19.5 \mathrm{~min}$ in the MED group, which were similar. We had 10 patients in each group with double-level LDH that resulted slightly higher average operation time compared with Kulkarni et al. [6]. However, there was significantly less hospital stay ( $5.0 \pm 0.9$ vs. $2.5 \pm 0.6$ days), less blood loss $(66.1 \pm 24.1 \mathrm{~mL}$ vs. $32.1 \pm 12.5 \mathrm{~mL})$, and earlier return to work after the discharge $(25.5 \pm 7.3$ days vs. $18.0 \pm 4.0$ days) in the MED group proves its benefits over OLD.

Bookwalter et al. reported that $40 \%$ of their patients returned to work in fewer than 5 weeks proving its cost-effectiveness [21]. Caspar et al. reported a mean return-to-work time of 18.6 weeks [22] and Perez-Cruet et al. reported a mean return-towork time of 17.6 days [23]. Our protocol was encouraging patients to resume work within 2 weeks following discharge from the hospital. Fujii et al. reported an interesting study in six health-care professionals who were operated for percutaneous endoscopic discectomy for LDH [24]. Postoperatively, five out of the six physician patients returned the original job within a week because they had clinical duties. The shortest duration to return to work was reported by a 63-year-old orthopedic surgeon resumed working in his clinic 2 days after the surgery because he had scheduled clinical duty. The longest duration to return to work occurred in general medicine resident who took almost 2 weeks for the sick leave because he did not have clinical duties. The mean duration for the returning to work was 5.8 days after the surgery. At final follow-up ranging from 6 to 30 months, all physician patients were working without any residual pain. In the hands of the authors, the full endoscopic transforaminal decompression surgery is the preferred surgical option and allowed early return to work. These all reports again emphasize that although MED has faster rate of returning to daily activity or work, it again depends on the need of urgency to work as well. Fujii et al. noticed same thing as one orthopedic surgeon returned to work due to their work and other physician returned to work after 2 weeks due to no pending work [24]. Similarly, if we discuss about laborers who were the primary study population in our series from a developing nation did not have any social security or health-care compensation, and their family was dependent on their daily earning for their living. This made them similar to pending work in case of a surgeon suggested by Fujii et al. in their report [24]. Therefore, we believe even a week earlier to return to their previous work in the MED group of patients would mean a lot for these laborers as they start earning earlier than the OLD group of patients. Such reports have not been published earlier, and therefore, we believe that our experience would guide further regarding choice of treatment option in laborers when they have to undergo for operation for LDH.

There may be a few limitations in this study. First is about the selection of patients, which were as per surgeon's preference in outpatient clinic. Therefore, there might be some selection bias while selecting patients for OLD or MED. Second, we have found less complications, especially regarding recurrent disc herniation in the series. However, we believe that our patients were from labor group and unless they had severe symptomatic disc herniation, they tend to ignore it. The patients who had reported recurrent disc were having significant symptoms, which were luckily managed conservatively.

\section{Conclusion}

We would like to suggest although clinical improvement after discectomy surgery in laborers is similar between the OLD and MED groups, MED proved as an effective alternative to OLD in laborers with respect to return to work earlier. Such studies may further throw light in differential management of laborer population with MED versus OLD.

\section{References}

1. Balagué F, Mannion AF, Pellisé F, Cedraschi C. Non-specific low back pain. Lancet 2012;379:482-91.

2. McCall IW.Lumbarherniated disks. Radiol Clin North Am 2000;38:1293-309.

3. Van Boxem K, Cheng J, Patijn J, Van Kleef M, Lataster A, Mekhail N, et al. 11. Lumbosacral radicular pain. Pain Pract 2010;10:339-58.

4. Yoke CO, Ann TK. Study of lumbar disc pathology among a group of dockworkers. Ann Acad Med Singap 1979;8:81-5.

5. Yadav RI, Long L, Yanming C. Comparison of the effectiveness and outcome of microendoscopic and open discectomy in patients suffering from lumbar disc herniation.Medicine (Baltimore) 2019;98:e16627.

6. Kulkarni AG, Bassi A, Dhruv A. Microendoscopic lumbar discectomy: Technique and results of 188 cases. Indian J Orthop 2014;48:81-7.
7. He J, Xiao S, WuZ, Yuan Z. Microendoscopic discectomy versus open discectomy for lumbar disc herniation: A meta-analysis. Eur Spine J2016;25:1373-81.

8. Rompe JD, Eysel P, Zöllner J, Heine J. Prognostic criteria for work resumption after standard lumbar discectomy. Eur Spine J 1999;8:132-7.

9. Singh T, Sharma S, Nagesh S. Socio-economic status scales updated for 2017. Int J Res Med Sci 2017;5:3264-7.

10. Hoy DG, Smith E, Cross M, Sanchez-Riera L, Buchbinder R, Blyth FM, et al. The global burden of musculoskeletal conditions for 2010: An overview of methods. Ann Rheum Dis 2014;73:982-9.

11. Rothoerl RD, Woertgen C, Brawanski A. When should conservative treatmentfor lumbar disc herniation be ceased and surgery considered? Neurosurg Rev 2002;25:162-5. 
12. Foley KT.Microendoscopic discectomy. Tech Neurosurg 1997;3:301-7.

13. Tullberg T, Isacson J, Weidenhielm L. Does microscopic removal of lumbar disc herniation lead to better results than the standard procedure? Results of a oneyear randomized study. Spine (Phila Pa 1976) 1993;18:24-7.

14. Muramatsu K, Hachiya Y, Morita C. Postoperative magnetic resonance imaging of lumbar disc herniation: Comparison of microendoscopic discectomy and Love's method. Spine (Phila Pa 1976) 2001;26:1599-605.

15. Schizas C, Tsiridis E, Saksena J. Microendoscopic discectomy compared with standard microsurgical discectomy for treatment of uncontained or large contained discherniations. Neurosurgery 2005; 57:357-60.

16. Shin DA, Kim KN, Shin HC, Yoon DH. The efficacy of microendoscopic discectomy in reducing iatrogenic muscle injury. J Neurosurg Spine 2008;8:3943.

17. Garg B, Nagraja UB, Jayaswal A. Microendoscopic versus open discectomy for lumbar disc herniation: A prospective randomised study. J Orthop Surg (Hong Kong) $2011 ; 19: 30-4$.

18. Bhatia PS, Chhabra HS, Mohapatra B, Nanda A, Sangodimath G, Kaul R. Microdiscectomy or tubular discectomy: Is any of them a better option for management of lumbar disc prolapse. J Craniovertebr Junction Spine
$2016 ; 7: 146-52$.

19. Brock M, Kunkel P, Papavero L. Lumbar microdiscectomy: Subperiosteal versus transmuscular approach and influence on the early postoperative analgesic consumption. Eur Spine J2008;17:518-22.

20. Mayer HM, Brock M. Percutaneous endoscopic discectomy: Surgical technique and preliminary results compared to microsurgical discectomy. J Neurosurg 1993;78:216-25.

21. Bookwalter JW 3rd, Busch MD, Nicely D. Ambulatory surgery is safe and effective in radicular disc disease. Spine (Phila Pa 1976) 1994;19:526-30.

22. Caspar W, Campbell B, Barbier DD, Kretschmmer R, Gotfried Y. The Caspar microsurgical discectomy and comparison with a conventional standard lumbar disc procedure. Neurosurgery 1991;28:78-86.

23. Perez-Cruet MJ, Foley KT, Isaacs RE, Rice-Wyllie L, Wellington R, Smith MM, et al. Microendoscopic lumbar discectomy: Technical note. Neurosurgery 2002;51:S129-36.

24. Fujii Y, Yamashita K, Sugiura K, Ishihama Y, Manabe H, Tezuka F, et al. Early return to activity after minimally invasive full endoscopic decompression surgery in medical doctors. J Spine Surg 2020;6:S294-9.

\section{Conflict of Interest: NIL} Source of Support: NIL

\section{How to Cite this Article}

Modi HN, Kunder T, Bhavsar N, Patel PR | Which is Better in Laborers? A Comparison Between Open and Micro Endoscopic Discectomy| Back Bone: The Spine Journal | October 2020March 2021; 1(1): 8-12. 